\title{
Physical parameters for subdwarf B stars with composite spectra ${ }^{\star}$
}

\author{
R. Aznar Cuadrado and C. S. Jeffery
}

Armagh Observatory, College Hill, Armagh BT61 9DG, Northern Ireland

Received 5 November 2001 / Accepted 16 January 2002

\begin{abstract}
New intermediate-resolution spectra have been obtained for a number of subdwarf B stars having both single and composite spectra. Physical parameters have been determined for the sdB stars and, in compositespectrum systems, their cool companions. For these binaries, we have developed a method which uses the blueoptical spectrum to determine the effective temperatures of both stars, the surface gravity of the hot stars and the radius ratio of the system. The surface gravity of the cool star is measured using the infrared calcium triplet. The surface gravities of these cool companions identify them as main-sequence stars with masses in the range 0.8$1.2 M_{\odot}$, confirming a previous energy distribution analysis. There is also evidence that the composite-spectrum sdBs are more helium-poor than single-spectrum sdBs.
\end{abstract}

Key words. stars: formation - stars: early-type - stars: subdwarfs - stars: fundamental parameters stars: binaries: spectroscopic

\section{Introduction}

Subdwarf B (sdB) stars are the most extreme of horizontal branch stars, being predominantly helium stars of approximately half a solar mass overlaid by a hydrogen-rich veneer (Heber 1986). Common in both our own galaxy (Green et al. 1986) and in giant ellipticals (Brown et al. 1997), they present a problem for stellar evolution theory: how does a red giant star dispose of its entire hydrogenrich envelope prior to core helium ignition?

From an initial supposition that sdB stars were predominantly single, models including enhanced mass-loss rates (e.g. D'Cruz et al. 1996), and white dwarf mergers (Iben 1990; Saio \& Jeffery 2000) have been investigated. However a significant fraction of sdB stars are known to have composite spectra (Ferguson et al. 1984; Allard et al. 1994; Jeffery \& Pollacco 1998), leading to suggestions of a binary fraction between $50 \%$ and $100 \%$. Recent radial velocity studies (Saffer et al. 2001) have identified three distinct groups: 1) single-spectrum sdBs with small or negligible velocity variations, 2) single-spectrum sdBs with large velocity variations and likely periods of hours to days and 3) composite-spectrum sdBs with small velocity variations and relatively long periods. Another recent investigation finds that the second group comprises some

Send offprint requests to: C. S. Jeffery,

e-mail: csj@star.arm.ac.uk

* Based on observations made with the Isaac Newton and William Herschel Telescopes.
$60 \pm 8 \%$ of all sdBs (Maxted et al. 2001). The clear conclusion is that binary evolution plays a significant rôle in the formation of sdB stars.

For the group (2) sdBs, the binary companion is invisible. Radial velocity and, in some cases, light curve studies will yield vital clues about the overall dimensions of these binary systems, and hence about their previous evolution. For group (3), dynamical information is less accessible although very careful observations over a long time base will be an important tool in this endeavour. Fortunately and by definition, the binary companion in a composite spectrum can be seen.

We have already examined the flux distributions for a number of binaries (Aznar Cuadrado \& Jeffery 2001: Paper I) and concluded that the companions are main sequence stars. This contradicted previous analyses (Allard et al. 1994; Jeffery \& Pollacco 1998) which suggested that the companions were overluminous. Therefore it is important to verify the results spectroscopically. Such an approach carries an additional bonus. The near-infrared triplet lines of ionized calcium are very strong in latetype stars and provide a sensitive diagnostic of surface gravity, providing the effective temperature and metallicity are known (Cohen 1979; Jones et al. 1984; Smith \& Drake 1987; Jørgensen et al. 1992). If both surface gravities and the radius ratio can be measured, the mass ratio can be determined directly and provides a very important tool for exploring the previous evolution of this group of sdB stars. 
Table 1. Instrumental configurations.

\begin{tabular}{|l|l|c|c|c|c|c|c|c|}
\hline Dates & Telesc. & Spec. & Grating & Dichroic & Slit & Detector & $R$ & $\lambda / \AA$ \\
\hline 1997 Sep. 4 & INT & IDS & R1200R & - & $1.6^{\prime \prime}$ & TEK3 & 5000 & $8000-8800$ \\
1997 Sep. 12, 13 & WHT & ISIS & R1200B & 5700 & $1.2^{\prime \prime}$ & TEK1 & 4000 & $4200-4650$ \\
& & & R600R & 5700 & $1.2^{\prime \prime}$ & TEK2 & 4300 & $8000-8850$ \\
1998 Oct. 3, 4 & WHT & ISIS & R1200B & 5300 & $1.2^{\prime \prime}$ & TEK1 & 4000 & $4200-4650$ \\
1999 Mar. 26, 27 & INT & IDS & R1200B & - & $1.2^{\prime \prime}$ & TEK5 & 2500 & $3800-4700$ \\
& & & R1200R & - & $1.2^{\prime \prime}$ & TEK5 & 5000 & $8000-8850$ \\
\hline
\end{tabular}

In this paper we introduce the methods used to analyse the spectra of composite-spectrum sdB stars, and present results for an initial sample. The methods are tested by providing independent analyses for a number of singlespectrum sdB stars.

\section{Observations and data reduction}

The observations used in this analysis have been collected from the William Herschel $(4.2 \mathrm{~m})$ and the Isaac Newton $(2.5 \mathrm{~m})$ Telescopes at the Roque de los Muchachos Observatory on La Palma between 1997 and 1999. Instrumental setups for each observing run are given in Table 1.

The stars in the current analysis were all selected because they had been observed with IUE and their flux distributions were well understood (Aznar Cuadrado 2001). The possibility that composite spectrum systems might be chance alignments has also been examined for these stars (ibid.). The sample included a number of stars known to have composite spectra or to have an infrared colour excess, as well as a comparable number considered to be "single-spectrum" systems. The log of observations is given in Table 2 .

All stellar spectra were bias subtracted, flat-fielded, sky subtracted and one-dimensional spectra were extracted using standard IRAF packages. Copper-argon-neon comparison spectra were used to provide a wavelength calibration corresponding to each stellar observation. Spectra were normalized with respect to the local continuum.

The blue spectra are dominated by the hydrogen Balmer series, which are both temperature and gravity sensitive. Other prominent features present in both single and composite-spectrum subdwarfs are the He I $\lambda 4388 \AA$ and He I $\lambda 4471 \AA$ lines, the magnesium doublet at $\lambda 4481 \AA$, the silicon triplet at $\lambda \lambda 4553,4568$ and $4575 \AA$, and the $\mathrm{C}$ II $\lambda 4267 \AA$ doublet, amongst others. The near-infrared spectra of composite sdBs are dominated by the calcium triplet at $\lambda \lambda 8498,8542$ and $8662 \AA$. There are many weaker unidentified features. Several broad hydrogen Paschen lines come from the hot subdwarf. These are effectively invisible because the subdwarf spectrum is swamped by the cool star. However, they have the effect of depressing the apparent continuum in this spectral range.
Table 2. Spectroscopic observations of single and composite sdB stars.

\begin{tabular}{|c|l|r|r|r|r|}
\hline Star & Telesc. & $\begin{array}{c}\text { HJD } \\
(-2450000)\end{array}$ & $\begin{array}{c}\lambda_{\text {cen }} \\
(\AA)\end{array}$ & $\begin{array}{c}\text { exp } \\
(\mathrm{s})\end{array}$ & $S / N$ \\
\hline PG 0004+133 & WHT & 705.704 & 4400 & 1200 & 80 \\
PG 0110+262 & WHT & 704.639 & 4400 & 1800 & 92 \\
& WHT & 705.733 & 8400 & 1800 & 74 \\
PG 0229+064 & WHT & 1091.702 & 4400 & 200 & 140 \\
PG 0240+046 & WHT & 1091.721 & 4400 & 850 & 140 \\
PG 0342+026 & WHT & 705.748 & 4400 & 900 & 93 \\
PG 0749+658 & INT & 1264.399 & 4250 & 300 & 70 \\
& INT & 1264.538 & 8400 & 600 & 48 \\
PG 0839+399 & INT & 1265.387 & 4250 & 600 & 50 \\
PG 1104+243 & INT & 1265.667 & 4250 & 300 & 52 \\
& INT & 1264.596 & 8400 & 300 & 65 \\
PG 1233+427 & INT & 1265.591 & 4250 & 150 & 60 \\
PG 1701+359 & WHT & 704.359 & 4400 & 900 & 150 \\
& WHT & 704.359 & 8400 & 900 & 73 \\
PG 1718+519 & WHT & 705.377 & 4400 & 1200 & 80 \\
& WHT & 705.378 & 8400 & 1200 & 60 \\
PG 2110+127 & WHT & 704.399 & 4400 & 1200 & 100 \\
& INT & 696.543 & 8400 & 900 & 90 \\
PG 2135+045 & WHT & 1090.366 & 4400 & 900 & 70 \\
& INT & 696.580 & 8400 & 1800 & 35 \\
PG 2148+095 & WHT & 705.427 & 4400 & 1200 & 170 \\
& WHT & 705.426 & 8400 & 1200 & 100 \\
PG 2259+134 & WHT & 704.454 & 4400 & 1800 & 65 \\
\hline
\end{tabular}

\section{Radial velocities}

The spectra in our sample included both single and composite systems. Their radial velocities were measured by cross-correlation with theoretical models for both hot and cool star spectra.

This process includes the subtraction of the continuum and the conversion of the wavelength scale to logarithmic units, before calculating the cross-correlation function (ccf). Several spectral regions were excluded from the ccf, including wavelengths corresponding to bad CCD columns, cool-star features in composite systems (e.g. the $G$-band at $\lambda 4200 \AA$ ), broad Balmer lines, or strong lines from the observed spectrum that didn't appear in the synthetic spectrum. The ccf was then converted to velocity units and the position of its peak was measured by fitting a Gaussian.

The cross-correlation templates used for measuring the radial velocities $v_{\text {rad }}$ of each observed spectrum were taken to be the best-fit model spectrum for the total system 
Table 3. Heliocentric radial velocity measurements of single and composite sdB stars.

\begin{tabular}{|c|c|c|c|c|}
\hline Star & $\begin{array}{c}\text { HJD } \\
(-2450000) \\
\end{array}$ & $\begin{array}{c}\mathrm{sdB} \\
v \\
\end{array}$ & $\begin{array}{c}\text { K star } \\
v \\
\end{array}$ & $\delta v$ \\
\hline \multicolumn{5}{|l|}{ Single } \\
\hline PG $0004+133$ & 705.704 & $-20.6 \pm 4$ & & \\
\hline PG $0229+064$ & 1091.702 & $7.6 \pm 4$ & & \\
\hline PG $0240+046$ & 1091.721 & $63.4 \pm 2$ & & \\
\hline PG $0342+026$ & 705.748 & $13.4 \pm 8$ & & \\
\hline PG 0839+399 & 1265.387 & $25.7 \pm 8$ & & \\
\hline PG $1233+427$ & 1265.591 & $65.5 \pm 8$ & & \\
\hline PG $2259+134$ & 704.454 & $-14.4 \pm 6$ & & \\
\hline \multicolumn{5}{|l|}{ Composite } \\
\hline PG $0110+262$ & $\begin{array}{l}704.639 \\
705.733\end{array}$ & $\begin{array}{l}1.0 \pm 6 \\
\end{array}$ & $\overline{36.9} \pm 2$ & 35.9 \\
\hline \multirow[t]{2}{*}{ PG 0749+658 } & 1264.399 & $-21.0 \pm 7$ & - & 0.2 \\
\hline & 1264.538 & - & $-21.2 \pm 2$ & \\
\hline \multirow[t]{2}{*}{ PG $1104+243$} & 1265.667 & $-4.6 \pm 8$ & - & 8.4 \\
\hline & 1264.596 & - & $-13.0 \pm 4$ & \\
\hline \multirow[t]{2}{*}{ PG $1701+359$} & 704.359 & $-121.9 \pm 3$ & - & 13.9 \\
\hline & 704.359 & & $-135.8 \pm 2$ & \\
\hline \multirow[t]{2}{*}{ PG $1718+519$} & 705.377 & $-63.3 \pm 3$ & 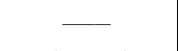 & 14.7 \\
\hline & 705.378 & - & $-48.6 \pm 1$ & \\
\hline \multirow[t]{2}{*}{ PG $2110+127$} & 704.399 & $27.0 \pm 1$ & - & 1.1 \\
\hline & 696.543 & - & $25.9 \pm 1$ & \\
\hline PG $2135+045$ & 1090.365 & $-30.0 \pm 1$ & $\overline{0}$ & 2.0 \\
\hline \multirow[t]{2}{*}{ PG $2148+095$} & 705.427 & $-152.7 \pm 1$ & $-20.0+2$ & 34.8 \\
\hline & 705.426 & - & $-117.9 \pm 2$ & \\
\hline
\end{tabular}

as described below. Hence, for single spectrum sdBs, we obtain a single radial velocity. For composite systems, the blue spectrum is dominated by lines from the hot star and so provides the sdB star velocity. The red spectrum is dominated by calcium lines from the cool companion and hence gives the cool star velocity.

The heliocentric velocities measured from each observation are given in Table 3. Errors on individual velocities are the formal errors given by the least squares Gaussian fit to the ccf peaks. The actual errors are probably much larger, but difficult to determine quantitatively (the typical ccf width is $\left.200-400 \mathrm{~km} \mathrm{~s}^{-1}\right)$. The standard deviation about the mean $\left(\sim 14 \mathrm{~km} \mathrm{~s}^{-1}\right)$ may be a better indication.

In composite systems, the difference $\delta v$ between the two component radial velocities would be a measure of the lower limit to the orbital velocity of the sdB star. Systems with large velocity differences between hot and cool components could be short-period systems, i.e. PG 0110+262, PG 1701+359, PG 1718+519 and PG 2148+095.

In the interim, other groups have used radial velocity studies to detect binary sdB stars (Maxted et al. 2001; Saffer et al. 2001). From our sample, the single-spectrum sdB PG $0839+399$ is a radial velocity binary (Maxted et al. 2001), while the composite-spectrum sdBs PG 0749+658 and PG $1701+359$ do not show detectable velocity variations (ibid.). Consequently, our conclusions may be subject to unidentified systematic errors. In the case of PG 0229+064 heliocentric radial velocities of $+8 \pm 2$ and
$+8 \pm 3 \mathrm{~km} \mathrm{~s}^{-1}$ measured on 1998 July 20 and September 13 respectively (Ramspeck et al. 2001) are essentially identical with our own measurement. Additional velocities for PG 1233+427 (53 $\pm 2 \mathrm{~km} \mathrm{~s}^{-1}$ : 2000 January 30 and 31), PG $0342+026$ (15 $\pm 2 \mathrm{~km} \mathrm{~s}^{-1}$ : 1998 Sep. 11, $14 \pm 2 \mathrm{~km} \mathrm{~s}^{-1}$ : 2000 Jan. 30) and PG0749+658 (-11 $\pm 2 \mathrm{~km} \mathrm{~s}^{-1}: 2000$ Jan. 31) have also been communicated to us (Edelmann \& Heber, private communication). These are not sufficiently different from our own measurements to make us suspect that any are short-period spectroscopic binaries.

Prior to analysis, the wavelengths of the observed spectra were corrected to the laboratory rest frame by applying these measured radial velocity shifts.

\section{Spectral analysis}

The aim of this study is to measure the various physical parameters, including effective temperature $\left(T_{\text {eff }}\right)$, surface gravity $(\log g$ ), chemical composition and, for binary stars, the radius ratio directly from optical and nearinfrared spectra. This is achieved by finding the best-fit model spectra within a grid of theoretical models using the method of $\chi^{2}$ minimization.

The methods for hot single stars have been described in detail (Jeffery et al. 2001), including the generation of model atmospheres (STERNE), the synthesis of model spectra (SPECTRUM) and the least-squares minimization (SFIT). These techniques have been extended to model binary stars which consist of both a hot and a cool component. Although they have already been introduced (Jeffery \& Aznar Cuadrado 2001), the method is described more formally here.

The fundamental assumption is that both cool and hot absorption sources are primarily stellar. We consider the observed normalized spectrum $S_{\lambda}$. Our aim is to reconstruct the best-fit model spectrum

$s_{\lambda}=\frac{\theta_{1}^{2} f_{\lambda 1}+\theta_{2}^{2} f_{\lambda 2}}{\theta_{1}^{2} f_{\mathrm{c} 1}+\theta_{2}^{2} f_{\mathrm{c} 2}}$

where $\theta$ are the stellar angular diameters, $f_{\lambda}$ is the theoretical emergent flux, $f_{\mathrm{c}}$ is the continuum flux and subscripts 1 and 2 refer to the individual stellar components. The fluxes are functions of each star's properties: $f_{1}=f_{1}\left(T_{\text {eff } 1}, \log g_{1}, \ldots\right)$ and $f_{2}=f_{2}\left(T_{\text {eff } 2}, \log g_{2}, \ldots\right)$. The angular diameters cannot be solved for explicitly from $S$, but their ratio gives $R_{2} / R_{1}=\theta_{2} / \theta_{1}$.

The principal free parameters which govern the measured spectrum are thus:

\footnotetext{
- $T_{\text {eff } 1}, T_{\text {eff } 2}$ : effective temperature;

- $\log g_{1}, \log g_{2}$ : surface gravity;

$-R_{2} / R_{1}$ : radius ratio;

- $v \sin i_{1}, v \sin i_{2}$ : rotational velocity;

- $v_{1}, v_{2}$ : radial velocity;

- $v_{\mathrm{t} 1}, v_{\mathrm{t} 2}$ : microturbulent velocity;
} 
- $y_{1}, y_{2}$ : helium abundance ${ }^{1}$

$-[\mathrm{Fe} / \mathrm{H}]_{1},[\mathrm{Fe} / \mathrm{H}]_{2}$ : metallicity ${ }^{2}$.

The radial velocities have already been discussed. Ideally, stellar composition entails many more free parameters than metallicity and helium abundance alone, but requires high-resolution spectroscopy to measure, as does the microturbulent velocity. Helium abundance cannot be measured directly for cool sources. Tests showed that $[\mathrm{Fe} / \mathrm{H}]_{2}$ cannot be uniquely determined from the given data. Similarly, given the magnitude of errors in $T_{\text {eff } 1}$, it is not practical to measure $[\mathrm{Fe} / \mathrm{H}]_{1}$ in detail. Thus the following assumptions are made. The abundances of all elements other than hydrogen and helium are in proportion to their cosmic abundances, with $[\mathrm{Fe} / \mathrm{H}]_{1}=[\mathrm{Fe} / \mathrm{H}]_{2}=0$. The helium abundance of the cool star is normal: $y_{2}=y_{\odot}$. The adopted microturbulent velocities are typical for earlytype stars $v_{\mathrm{t} 1}=5 \mathrm{~km} \mathrm{~s}^{-1}$ and main-sequence late-type stars $v_{\mathrm{t} 2}=2 \mathrm{~km} \mathrm{~s}^{-1}$. The latter assumption is very important as it affects both the metallicity $[\mathrm{Fe} / \mathrm{H}]_{2}$ (see above) and the derived radius ratio $R_{2} / R_{1}$. We have adopted $v_{\mathrm{t} 2}=2 \mathrm{~km} \mathrm{~s}^{-1}$ in order that the latter quantity as derived from spectral fitting be as consistent as possible with $\theta_{2} / \theta_{1}$ derived from spectrophotometry (Paper I) where we used cool star models computed with $v_{\mathrm{t}_{2}}=2 \mathrm{~km} \mathrm{~s}^{-1}$. Secondary effects on $T_{\text {eff } 2}$ and $\log g_{2}$ are not significant here.

\subsection{Model grids}

The model atmospheres and flux distributions used to analyse the hot star were computed with the lineblanketed plane-parallel LTE code sTERNE. The highresolution spectra were calculated with the LTE code SPECTRUM (see Jeffery et al. 2001).

The model atmospheres were calculated on a three-dimensional rectangular grid defined by $T_{\text {eff }}=$ $18000(2000) 40000 \mathrm{~K}, \log g=4.5(0.5) 7.0$, and composition $n_{\mathrm{H}}=1-n_{\mathrm{He}}, n_{\mathrm{He}}=0.01,0.10(0.10) 0.60$ and $[\mathrm{Fe} / \mathrm{H}]=0$. The larger value of $n_{\mathrm{H}}$ or $n_{\mathrm{He}}$ is reduced to compensate for trace elements.

Synthetic spectra were calculated on wavelength intervals 3800-5020 $\AA$ (blue) and 8450-8670 $\AA$ (CaT). Linelists comprising some 142 absorption lines of hydrogen, helium, carbon, magnesium and silicon were taken from the list of assessed data for hot stars LTE_LINES (Jeffery 1991). Microturbulent velocity $v_{\mathrm{t}}=5.0 \mathrm{~km} \mathrm{~s}^{-1}$ and solar abundances for all elements other than hydrogen and helium were assumed (see above).

\footnotetext{
1 The helium abundance is measured from models in which the fractional abundance by number $n_{\mathrm{He}}$ is specified. Several authors give the number ratio $y=n_{\mathrm{He}} / n_{\mathrm{H}}$ instead. We give the latter in Tables 4 and 5 and elsewhere.

2 Providing the stars are hydrogen-rich, the metallicity $([\mathrm{Fe} / \mathrm{H}])$ represents the logarithm of the value relative to the solar metal abundance, assuming a solar distribution of elements heavier than helium.
}

Model atmospheres and flux distributions used to analyse the cool star were taken from the Kurucz' standard grid of ATLAS models (Kurucz 1993a), for $T_{\text {eff }}=$ $3500(500) 8000, \log g=2.0(0.5) 4.5,[\mathrm{Fe} / \mathrm{H}]=-0.5,-0.3$, 0.0 and $v_{\mathrm{t}}=2.0 \mathrm{~km} \mathrm{~s}^{-1}$.

Grids of high resolution spectra were calculated in the same spectral regions as for the hot star using Kurucz' code SYNTHE (Kurucz 1993b; Jeffery et al. 1996) assuming a microturbulent velocity $v_{\mathrm{t}}=2 \mathrm{~km} \mathrm{~s}^{-1}$.

\subsection{SFIT}

For a given observation, an optimum fit was obtained by minimizing $\chi^{2}$,

$\chi^{2}=\sum_{\lambda} \frac{\left(S_{\lambda}-s_{\lambda}\right)^{2}}{\sigma_{\lambda}^{2}}$

the weighted square residual between the normalized observed spectrum, $S_{\lambda}$, and the theoretical spectrum,

$s_{\lambda}^{\prime}=s_{\lambda}\left(T_{\mathrm{eff}}, \log g\right) \otimes I(\Delta \lambda) \otimes V(v \sin i, \beta)$,

where $I(\Delta \lambda)$ and $V(v \sin i, \beta)$ represent the instrumental and rotational broadening, respectively. Instrumental broadening is measured from the width of the emission lines in the copper-argon comparison lamp. The $\chi^{2}$ minimization was carried out using a variant of the algorithm Amoeba (Press et al. 1989; Jeffery et al. 2001).

In the construction of $\chi^{2}$, each wavelength point was given a weight $w_{\lambda}=1 / \sigma_{\lambda}$, the inverse of the standard deviation of the mean normalized flux in line-free regions. In our spectra $\sigma_{\lambda} \sim 0.01$. Some spectral regions needed to be excluded from the fit (e.g. bad columns or strong lines missing from the theoretical spectrum). In SFIT, such defects are masked by increasing $\sigma$ in appropriate wavelength intervals. We used $\sigma_{\lambda}=0.1$.

In any such fitting procedure, the normalization of the observed spectrum can be of crucial importance (Jeffery 1998). The problem is to normalize the observed spectrum correctly without, for example, compromising the wings of broad absorption lines. This is particularly difficult when there is an unknown contribution to the line opacity from metal lines in a cool star companion, so that there may be no "true" continuum anywhere in the observed spectrum.

The initial normalization was performed by fitting a low-order spline function to a series of pseudo-continuum points, usually the highest points in the spectrum. SFIT includes two re-normalization algorithms which may be used to optimize the fit (cf. Jeffery et al. 1998). One computes a low-order polynomial fit to the residual, the other applies a low-pass Gaussian filter. A second order polynomial was used to renormalize the spectrum in wavelength ranges 4200-4650 $\AA$ and 8000-8850 $\AA$ while a third order polynomial was used in the region of high order Balmer lines $\left(\mathrm{H}_{9}, \mathrm{H}_{8}, \mathrm{H}_{\epsilon}\right.$, etc. $)$. Because low-order polynomials are used, individual line profiles are unaffected.

In principle and for suitable data with negligible noise, SFIT can solve simultaneously for as many parameters as 
Table 4. Atmospheric properties of single-spectrum sdB stars measured spectroscopically using SFIT and previously.

\begin{tabular}{|l|c|c|c|l|}
\hline Star & $T_{\text {eff }}$ & $\log g$ & $y$ & Reference \\
\hline PG 0004+133 & ${ }^{\dagger} 25025 \pm 400$ & $4.97 \pm 0.10$ & $0.01 \pm 0.01$ & SFIT \\
& $25025 \pm 400$ & & & Paper I \\
& $24700 \pm 1300$ & $4.5 \pm 0.2$ & 0.028 & Moehler et al. (1990a) \\
\hline PG 0229+064 & $18000 \pm 1025$ & $4.35 \pm 0.10$ & $0.33 \pm 0.01$ & SFIT \\
& $20100 \pm 400$ & & & Paper I \\
& $19000 \pm 950$ & $4.55 \pm 0.10$ & 0.16 & Ramspeck et al. (2001) \\
& $22000 \pm 1000$ & $4.65 \pm 0.15$ & 0.137 & Heber et al. (1999) \\
\hline PG 0240+046 & $36200 \pm 400$ & $6.25 \pm 0.10$ & $1.94 \pm 0.02$ & SFIT \\
& $34800 \pm 1850$ & & & Paper I \\
& $37000 \pm 2000$ & $5.3 \pm 0.3$ & 1.222 & Thejll et al. (1994) \\
\hline PG 0342+026 & $24000 \pm 375$ & $5.17 \pm 0.10$ & $0.01 \pm 0.01$ & SFIT \\
& $27900 \pm 975$ & & & Paper I \\
& $24000 \pm 1200$ & $4.90 \pm 0.20$ & 0.003 & Heber et al. (1999) \\
& $25000 \pm 2500$ & $5.25 \pm 0.20$ & 0.000 & Theissen et al. (1995) \\
& $26220 \pm 1000$ & $5.67 \pm 0.15$ & 0.004 & Saffer et al. (1994) \\
& $22300 \pm 1000$ & $5.00 \pm 0.30$ & 0.000 & Lamontagne et al. (1987) \\
\hline PG 0839+399 & $37300 \pm 500$ & $6.02 \pm 0.10$ & $<0.01 \pm 0.01$ & SFIT \\
& $35600 \pm 1800$ & & & Paper I \\
& $36100 \pm 1000$ & $5.91 \pm 0.15$ & 0.002 & Saffer et al. (1994) \\
\hline PG 1233+426 & $25560 \pm 550$ & $5.52 \pm 0.10$ & $<0.01 \pm 0.01$ & SFIT \\
& $28750 \pm 900$ & & & Paper I \\
& $26500 \pm 1000$ & $5.60 \pm 0.15$ & 0.005 & Saffer et al. (1994) \\
& $26200 \pm 1500$ & $5.30 \pm 0.30$ & 0.000 & Lamontagne et al. (1985) \\
\hline PG 2259+134 & $28500 \pm 600$ & $5.93 \pm 0.10$ & $0.02 \pm 0.01$ & SFIT \\
& $28350 \pm 750$ & & & Paper I \\
& $22500 \pm 2500$ & $5.00 \pm 0.20$ & 0.000 & Theissen et al. (1995) \\
& $28500 \pm 1600$ & $5.30 \pm 0.20$ & 0.022 & Theissen et al. (1993) \\
\hline
\end{tabular}

$\dagger T_{\text {eff }}$ assumed from Paper I.

required. In practice, it is necessary to restrict the free solution to between two and three parameters at a time, keeping others fixed, and to iterate until the optimum solution is obtained. SFIT requires a set of initial estimates for the free parameters. Results from the flux distribution analysis (Paper I) were used for $T_{\text {eff } 1}, T_{\text {eff } 2}$ and the radius ratio. Standard values were assumed for $\log g_{1}, \log g_{2}$ and $y_{1}$.

\subsection{Analysis: single stars}

For single sdB stars, SFIT was applied to the blue spectra. The first parameter derived was the composition $y$ of the $\mathrm{sdB}$ star. Within the $T_{\text {eff }}$ range of sdB stars, the strength of helium lines depends far more on helium abundance than $T_{\text {eff }}$ or $\log g . T_{\text {eff }}$ and $\log g$ were found next by finding the best fit to the Balmer lines. Rotational broadening is small compared with the instrumental profiles in these spectra.

Table 4 presents the results of the spectral analysis of single sdB stars (labeled SFIT), together with the results of the flux distribution analysis (Paper I) and results from literature.

\subsection{Analysis: binary stars}

For composite sdB stars, SFIT was applied separately to both blue and red spectra. Again, the first parameter to be fixed from the analysis of the blue spectrum is the compo- sition of the sdB star, i.e. $y_{1}$. Afterwards, $T_{\text {eff } 1}, \log g_{1}$ of the sdB star and $R_{2} / R_{1}$ are found by fitting the observed Balmer lines. The lower limit of the model grid was occasionally too large to fit the observed helium lines. In these cases only an upper limit to $y_{1}$ can be given.

The radius ratio $R_{2} / R_{1}$ is directly related to the effective temperatures of both components, so must be a "free" parameter whenever either $T_{\text {eff }}$ is free. The contribution of the cool companion in the blue spectrum is reflected in the presence and strength of some metallic lines, being good indicators of the temperature of the cool star and the radius ratio of the system. Therefore, the blue spectrum is also used to fix $T_{\text {eff } 2}$ and $R_{2} / R_{1}$. It was frequently difficult to find a solution for $T_{\text {eff } 1}, T_{\text {eff } 2}$ and $R_{2} / R_{1}$ consistent with the flux distribution analysis (Paper I). In cases of conflict, we attempted to keep $R_{2} / R_{1}$ consistent between the two studies, although even this was not always possible (Table 5).

In the initial analysis of the blue spectrum, the cool companion is assumed to have $\log g_{2}=4.5$ (cf. Paper I). Applying SFIT gives new values, first for $y_{1}$, then for $T_{\text {eff } 1}$, $\log g_{1}, T_{\text {eff } 2}$ and $R_{2} / R_{1}$.

With these improved estimates for the sdB star properties, the red spectrum is analyzed. In particular, $\log g_{2}$ is determined by fitting the calcium triplet.

A second analysis is now performed in the blue in order to refine the fit to the hot star spectrum, using the 
Table 5. Atmospheric properties of composite sdB stars measured spectroscopically using SFIT and previously. Subscript 1 refers to the hot subdwarf, subscript 2 refers to the cool companion.

\begin{tabular}{|c|c|c|c|c|c|c|c|}
\hline Star & $T_{\text {eff } 1}$ & $\log g_{1}$ & $y_{1}$ & $T_{\text {eff } 2}$ & $\log g_{2}$ & $R_{2} / R_{1}$ & Ref. $^{\dagger}$ \\
\hline PG 0110+262 & $\begin{array}{c}21000 \pm 750 \\
21050 \pm 575 \\
21000 \pm 1000 \\
22000 \pm 1000 \\
22000 \pm 1500\end{array}$ & $\begin{array}{l}5.17 \pm 0.17 \\
<5.90 \pm 0.10 \\
<5.50 \pm 0.10\end{array}$ & $<0.01 \pm 0.01$ & $\begin{array}{l}5250 \pm 800 \\
5485 \pm 200 \\
5000 \pm 500 \\
5500 \pm 500 \\
4500 \pm 500\end{array}$ & $4.53 \pm 0.21$ & $\begin{array}{c}3.2 \pm 1.9 \\
4.2 \pm 0.2 \\
6.0 \\
4.4 \\
7.8\end{array}$ & $\begin{array}{l}\text { SFIT } \\
1 \\
2 \\
3 \\
4\end{array}$ \\
\hline PG 0749+658 & $\begin{array}{c}25400 \pm 500 \\
25050 \pm 675 \\
24600 \pm 1000 \\
23500 \pm 1500\end{array}$ & $\begin{array}{l}5.70 \pm 0.11 \\
5.54 \pm 0.15\end{array}$ & $\begin{array}{c}<0.01 \pm 0.02 \\
0.004\end{array}$ & $\begin{array}{l}5000 \pm 500 \\
5600 \pm 300 \\
4125 \pm 500\end{array}$ & $4.58 \pm 0.24$ & $\begin{array}{c}3.5 \pm 1.2 \\
3.9 \pm 0.3 \\
6.3\end{array}$ & $\begin{array}{l}\text { SFIT } \\
1 \\
5^{*} \\
4\end{array}$ \\
\hline PG 1104+243 & $\begin{array}{c}32850 \pm 1550 \\
28000 \pm 875 \\
27500 \pm 1500 \\
27200 \pm 1500 \\
28000 \pm 5000\end{array}$ & $\begin{array}{l}5.40 \pm 0.12 \\
5.50 \pm 0.30\end{array}$ & $0.01 \pm 0.02$ & $\begin{array}{c}6400 \pm 1000 \\
5735 \pm 150 \\
4300 \pm 500 \\
4600 \pm 1000\end{array}$ & $4.30 \pm 0.31$ & $\begin{array}{c}5.9 \pm 1.1 \\
6.1 \pm 0.2 \\
10.6 \\
9.8\end{array}$ & $\begin{array}{l}\text { SFIT } \\
1 \\
4 \\
6 \\
7\end{array}$ \\
\hline PG $1701+359$ & $\begin{array}{c}32500 \pm 1325 \\
36075 \pm 700 \\
30000 \pm 2500 \\
28500 \pm 1500 \\
26250 \pm 1250\end{array}$ & $\begin{array}{l}5.75 \pm 0.12 \\
5.00 \pm 0.20 \\
5.80 \pm 0.20\end{array}$ & $\begin{array}{c}<0.01 \pm 0.01 \\
0.000\end{array}$ & $\begin{array}{c}6000 \pm 1000 \\
6450 \pm 230 \\
4000 \pm 500\end{array}$ & $4.60 \pm 0.23$ & $\begin{array}{c}2.7 \pm 1.8 \\
4.8 \pm 0.2 \\
6.2\end{array}$ & $\begin{array}{l}\text { SFIT } \\
1 \\
8 \\
4 \\
9\end{array}$ \\
\hline PG $1718+519$ & $\begin{array}{l}29000 \pm 1550 \\
29950 \pm 1100 \\
30000 \pm 2500 \\
25000 \pm 1500 \\
23300 \pm 1000\end{array}$ & $\begin{array}{l}6.00 \pm 0.14 \\
5.00 \pm 0.20 \\
4.25 \pm 0.20\end{array}$ & $\begin{array}{c}<0.01 \pm 0.01 \\
0.000\end{array}$ & $\begin{array}{c}5200 \pm 400 \\
5925 \pm 70 \\
5125 \pm 500 \\
4300 \pm 500\end{array}$ & $4.55 \pm 0.23$ & $\begin{array}{c}4.8 \pm 1.6 \\
8.2 \pm 0.3 \\
10.7\end{array}$ & $\begin{array}{l}\text { SFIT } \\
1 \\
8 \\
4 \\
9\end{array}$ \\
\hline $\begin{array}{l}\text { PG 2110+127 } \\
\text { (Fig. 2) }\end{array}$ & $\begin{array}{l}26500 \pm 1700 \\
24900 \pm 6500 \\
30000 \pm 2500 \\
33700 \pm 1000 \\
26000 \pm 1500\end{array}$ & $\begin{array}{l}5.20 \pm 0.18 \\
5.00 \pm 0.20 \\
5.33 \pm 0.15\end{array}$ & $\begin{array}{c}<0.01 \pm 0.06 \\
0.000 \\
0.004\end{array}$ & $\begin{array}{l}5400 \pm 400 \\
5500 \pm 575 \\
5375 \pm 500 \\
4500 \pm 500\end{array}$ & $4.40 \pm 0.24$ & $\begin{array}{l}4.7 \pm 1.1 \\
5.5 \pm 0.3\end{array}$ & $\begin{array}{l}\text { SFIT } \\
1 \\
8 \\
5 \\
4\end{array}$ \\
\hline PG 2135+045 & $\begin{array}{c}28400 \pm 800 \\
26325 \pm 9950 \\
32100 \pm 1000 \\
27000 \pm 1500\end{array}$ & $\begin{array}{l}4.80 \pm 0.22 \\
4.79 \pm 0.15\end{array}$ & $\begin{array}{c}<0.01 \pm 0.01 \\
0.016\end{array}$ & $\begin{array}{c}5000 \pm 500 \\
4375 \pm 1790 \\
4400 \pm 500\end{array}$ & $4.40 \pm 0.30$ & $\begin{array}{c}3.1 \pm 0.5 \\
4.7 \pm 0.6 \\
6.5\end{array}$ & $\begin{array}{l}\text { SFIT } \\
1 \\
5^{*} \\
4\end{array}$ \\
\hline $\begin{array}{l}\text { PG 2148+095 } \\
\text { (Fig. 4) }\end{array}$ & $\begin{array}{c}30000 \pm 860 \\
22950 \pm 825 \\
25000 \pm 1000 \\
26000 \pm 1500\end{array}$ & $\begin{array}{l}4.90 \pm 0.16 \\
<5.80 \pm 0.10\end{array}$ & $<0.01 \pm 0.01$ & $\begin{array}{l}5700 \pm 400 \\
4375 \pm 200 \\
5000 \pm 500 \\
4300 \pm 500\end{array}$ & $4.40 \pm 0.31$ & $\begin{array}{c}3.0 \pm 0.8 \\
5.0 \pm 0.2 \\
6.0 \\
7.9\end{array}$ & $\begin{array}{l}\text { SFIT } \\
1 \\
2 \\
4\end{array}$ \\
\hline
\end{tabular}

${ }^{\dagger}$ References: 1 = Paper I; $2=$ Ulla \& Thejll (1998); $3=$ Thejll et al. (1995); $4=$ Allard et al. (1994);

$5=$ Saffer et al. (1994); $6=$ Lamontagne et al. (1987); $7=$ Ferguson et al. (1984); 8 = Theissen et al. (1995);

$9=$ Theissen et al. (1993).

* Saffer et al. (1994) did not recognise the composite nature of these stars.

new parameters for the cool star. The above procedure is repeated until the solutions converge.

Table 5 presents the results for composite spectrum sdB stars, together with previous results from the literature. Solar metallicity was adopted for all stars except PG $1104+243$, for which we adopted $[\mathrm{Fe} / \mathrm{H}]_{2}=-0.5$. The instrumental profile is large compared with any rotational broadening except in the cases of PG 1701+359 and PG $1718+519$, where $v \sin i=5$ and $10 \mathrm{kms}^{-1}$, respectively, were adopted.

Table 5 includes values for $R_{2} / R_{1}$ for some previous papers. These have been computed from cited flux ratios at $5500 \AA$, effective temperatures and/or spectral types and an appropriate bolometric correction.

\subsection{Errors}

The formal errors associated with the best fit parameters $x_{i}$ are given by the diagonal elements $\delta_{i}=\left(\alpha^{-1}\right)_{i i}$ of the inverse of the covariance matrix $\alpha$, whose elements are given by

$\alpha_{i j}=\sum_{\lambda}\left(\frac{\partial s_{\lambda}}{\partial x_{i}} \frac{\partial s_{\lambda}}{\partial x_{j}} / \sigma_{\lambda}^{2}\right)$ 
Table 6. Comparison of BINFIT and SFIT for a test binary.

\begin{tabular}{|l|c|c|c|}
\hline Parameter & Model & BINFIT & SFIT \\
\hline$T_{\text {eff }}(\mathrm{K})$ & 24000 & $24060 \pm 260$ & $24190 \pm 300$ \\
$\log g_{1}$ & 6.0 & & $6.02 \pm 0.05$ \\
$y_{1}$ & 0.10 & & $0.11 \pm 0.01$ \\
$T_{\text {eff } 2}(\mathrm{~K})$ & 4500 & $4550 \pm 150$ & $4500 \pm 200$ \\
$\log g_{2}$ & 4.5 & & $4.54 \pm 0.05$ \\
$R_{2} / R_{1}$ & 6.29 & $5.90 \pm 0.16$ & $6.27 \pm 0.20$ \\
\hline
\end{tabular}

Since SFIT is never run with all parameters free, the full covariance matrix is never computed. Thus the total errors $\sigma_{i}$ need to be obtained from a careful analysis of the partial errors $\delta_{i}$.

For a single-star spectrum, only the derivatives between $T_{\text {eff }}, \log g$ and $y$ need to be calculated. In the case of a binary system, the derivatives between the physical parameters of both components of the system are required. These have to be evaluated numerically, e.g.

$\frac{\partial x_{1}}{\partial x_{2}}=\frac{x_{1}^{\prime}-x_{1}}{\Delta x_{2}}$,

where $x_{1}$ represents parameters of the sdB star and $x_{2}$ represents parameters of the cool star. $\Delta x_{2}$ is an increment used to compute a change in $x_{1}$, usually $1 \%$ of $x_{2} . x_{1}^{\prime}$ are parameters derived when using $x_{2}+\Delta x_{2}$ as input to the fit.

The errors given in Tables 4 and 5 are total errors. For example the total error $\sigma$ in $T_{\text {eff } 1}$ is given by

$$
\begin{aligned}
\sigma_{T_{\mathrm{eff}_{1}}}^{2}= & \delta_{T_{\mathrm{eff}_{1}}^{2}}+\left(\frac{\partial T_{\mathrm{eff} 1}}{\partial \log g_{1}}\right)^{2} \delta_{\log g_{1}}^{2} \\
& +\left(\frac{\partial T_{\text {eff } 1}}{\partial y_{1}}\right)^{2} \delta_{y_{1}}^{2}+\left(\frac{\partial T_{\text {eff } 1}}{\partial \log g_{2}}\right)^{2} \delta_{\log g_{2}}^{2} \\
& +\left(\frac{\partial T_{\text {eff } 1}}{\partial\left(R_{2} / R_{1}\right)}\right)^{2} \delta_{R_{2} / R_{1}}^{2} .
\end{aligned}
$$

In addition to the formal errors cited, there are additional systematic errors. Principal amongst these are in the metallicity of the cool star, $[\mathrm{Fe} / \mathrm{H}]_{2}$. This has a strong influence on the measurement of $\log g_{2}$ from the calcium triplet, and hence on the radius ratio $R_{2} / R_{1}$ because the latter is primarily fixed by the strength of the metallines relative to Balmer lines in the blue spectrum. Highresolution spectra will be required to address this problem.

There are also systematic differences between the methods used to obtain $R_{2} / R_{1}$ in this paper and in Paper I. To compare these methods we have constructed a simple test. The energy distribution and normalized spectrum of a binary system containing a typical sdB star and a main-sequence star were computed. These were resampled to mimic the observational data available to us in each investigation. The data were then analyzed using BINFIT (to fit the flux distribution, Paper I) and SFIT independently. The model parameters and the results of the $\chi^{2}$ analysis are shown in Table 6 . The results are all consistent with the test model except the value of $R_{2} / R_{1}$ given by BINFIT. The errors associated with this parameter are
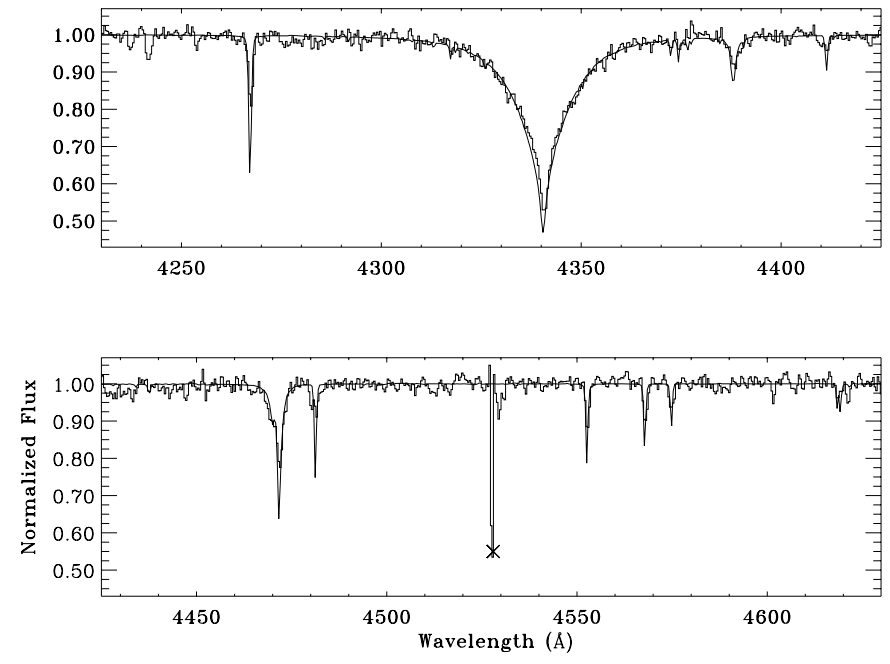

Fig. 1. Normalized blue spectrum of the single sdB star PG 0004+133 (histogram) together with the best fit model spectrum (polyline). The symbol $\times$ marks a CCD defect.

possibly underestimated, since they are only derived from the formal error in the angular diameters.

\section{Results}

\subsection{Single-star spectra}

Figure 1 shows the best fit model for the single sdB star PG 0004+133. In addition to results reported in Table 4, the following individual remarks are noted.

PG 0004+133: since only the $\mathrm{H} \gamma$ line is available, we have assumed $T_{\text {eff }}$ from Paper I.

PG 0229+064: with $y=0.33$, this is a helium-rich sdB star (Heber et al. 1999). The metal lines imply a higher metal abundance than assumed in the model. This has also been found by Ramspeck et al. (2001) who, in particular, find $\mathrm{C}$ and $\mathrm{N}$ overabundant by nearly one dex.

PG 0240+046: an even more helium-rich sdB star with $66 \%$ of He abundance, consistent with a previous abundance of $55 \%$ Thejll et al. (1994).

PG 0342+026: C, Si and Mg appear to be underabundant relative to the assumed solar composition.

PG0839+399 and PG1233+426: the helium abundance is below the measurement threshold, and metals are underabundant.

PG 2259+134: C appears to be underabundant.

\subsection{Composite spectra}

Results for sdB stars with composite spectra are shown in Table 5. Figures 2 and 3 show best fits for the composite $\mathrm{sdB}$ star, $\mathrm{PG} 2110+127$. The radius ratios $\left(R_{2} / R_{1}\right)$ and hence, by implication, the radii of the cool stars $\left(R_{2}\right)$ are all smaller than measured previously.

In nearly all cases, the He I lines are weaker than predicted by models with $n_{\mathrm{He} 1}=0.01$, implying hot star He abundances below this value. In addition, metal lines 


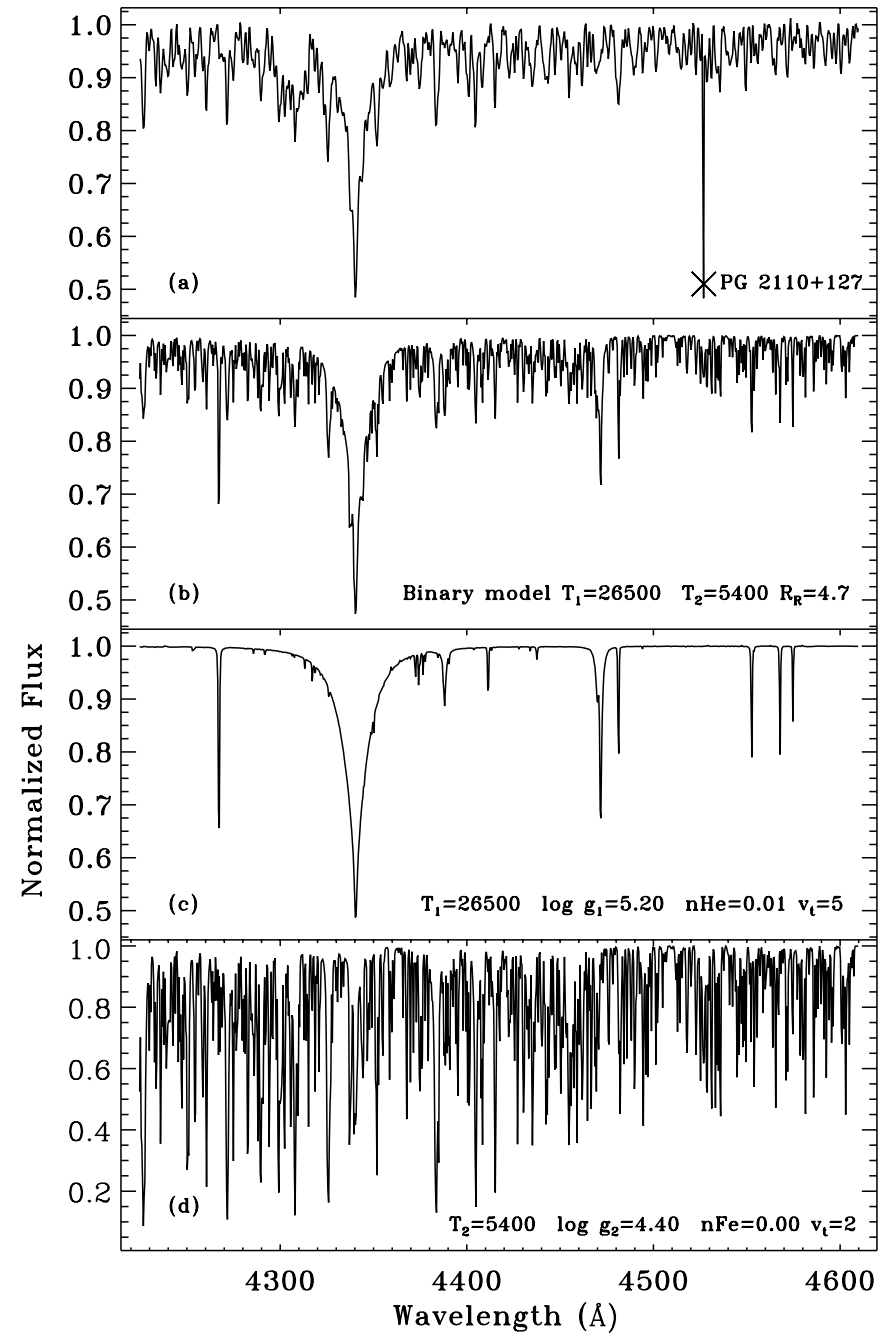

Fig. 2. Normalized blue spectrum of the composite PG $2110+127$ a) together with the best fit composite model spectrum b) formed by adding models with c) $T_{\text {eff } 1}=26500 \mathrm{~K}, \log g_{1}=5.20, y=0.01, v_{\mathrm{t} 1}=5 \mathrm{~km} \mathrm{~s}^{-1}$ and d) $T_{\text {eff } 2}=5400 \mathrm{~K}, \log g_{2}=4.40,[\mathrm{Fe} / \mathrm{H}]=0.00$, $v_{\mathrm{t} 2}=2 \mathrm{~km} \mathrm{~s}^{-1}$ with radius ratio $R_{2} / R_{1}=4.7$. The model spectra have been velocity shifted and degraded to match the observations. The symbol $\times$ marks a CCD defect.

from the hot star, (e.g. silicon, carbon and magnesium), appear to be too strong in the model compared with the observations. Since we assumed $[\mathrm{Fe} / \mathrm{H}]_{1}=0.0$, this implies that metals are generally underabundant in the $\mathrm{sdB}$ stars in our sample. This requires confirmation from highresolution spectroscopy. In addition to results reported in Table 5, the following individual remarks are noted.

PG 0110+262 and PG 0749+658: C underabundant

PG 1104+243: with $y=0.01 \pm 0.01$, this is the most helium-rich composite sdB in our sample. The strength of the $\mathrm{Ca} \mathrm{K}$ line and other metallic lines in the blue spectrum indicates $T_{\text {eff } 2}=6400 \mathrm{~K}, \log g_{2}=4.3$, and $R_{2} / R_{1} \sim 6$. However, assuming the same radius ratio and $[\mathrm{Fe} / \mathrm{H}]_{2}=0.0$, the red spectrum gives $T_{\text {eff } 2}=4500 \mathrm{~K}$ and $\log g_{2}=4$.6. Since the blue spectrum provides very strong constraints on $T_{\text {eff } 2}$ and $R_{2} / R_{1}$, it was necessary to adopt

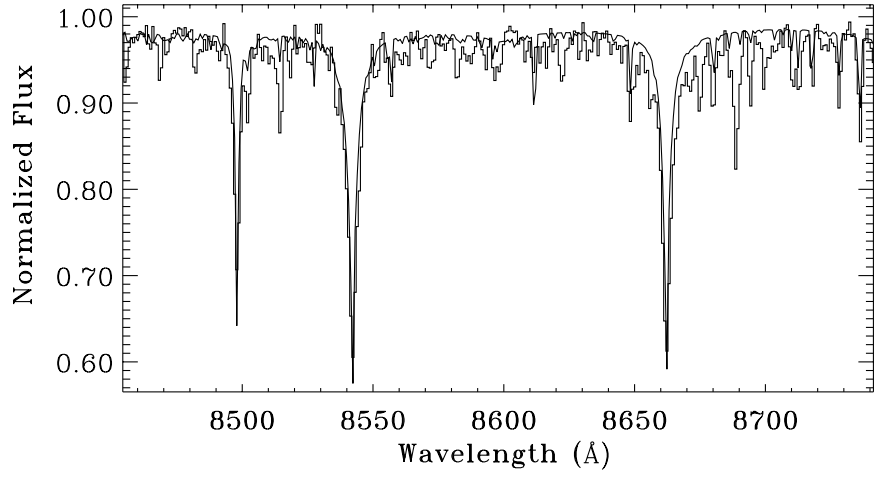

Fig. 3. Normalized red spectrum of the composite PG 2110+127 around the infrared calcium triplet (histogram) together with the best fit model spectrum (polyline).

a reduced value for $[\mathrm{Fe} / \mathrm{H}]_{2}=-0.5$ to maintain consistency with Paper I.

PG 1701+359 and PG 1718+519: C, Mg and Si underabundant. $R_{2} / R_{1}$ significantly smaller than in Paper I. This could be due to the adoption of too high metallicity $[\mathrm{Fe} / \mathrm{H}]_{2}$.

\section{PG 2110+127: C, Mg and Si underabundant.}

PG 2135+045: C, $\mathrm{Mg}$ and Si underabundant. $R_{2} / R_{1}$ significantly smaller than in Paper I. This is probably due to the absence of IUE LW and JHK photometry which led to particularly large errors in the Paper I $T_{\text {eff }}$ measurements.

PG 2148+095: C, $\mathrm{Mg}$ and $\mathrm{Si}$ underabundant. $R_{2} / R_{1}$ significantly smaller than in Paper I probably due to significant differences in $T_{\text {eff }}$. The latter are probably due to the absence of an IUE LW spectrum and a possible anomaly in the $J$-band photometry.

Significant differences between the results of the spectroscopic (SFIT) and photometric (Paper I) analyses have been discussed above. Tables 4 and 5 also include the results of earlier photometric and spectroscopic analyses. The current results agree well with previous spectroscopic analyses (Moehler et al. 1990a; Saffer et al. 1994; Heber et al. 1999) in the cases of PG 0342+026, PG 0839+399, PG $1233+426$ and PG 0749+658. They do not agree well in the cases of PG 0004+133, PG 0229+064, PG 2110+127 and PG $2135+045$.

The high helium abundance may contribute to the $T_{\text {eff }}$ discrepancy in PG 0229+064, a cool He-rich subdwarf with a relatively low surface gravity. Saffer et al. (1994) did not recognize the composite nature of $\mathrm{PG} 0749+658$ and PG 2135+045, and it is not clear how they modelled the spectrum of PG $2110+127$. When deriving the sdB parameters, Theissen et al. $(1993,1995)$ corrected for the continuum light of the cool companions, but not the (weaker) Balmer lines from the cool stars. Therefore these results may not be fully reliable. 

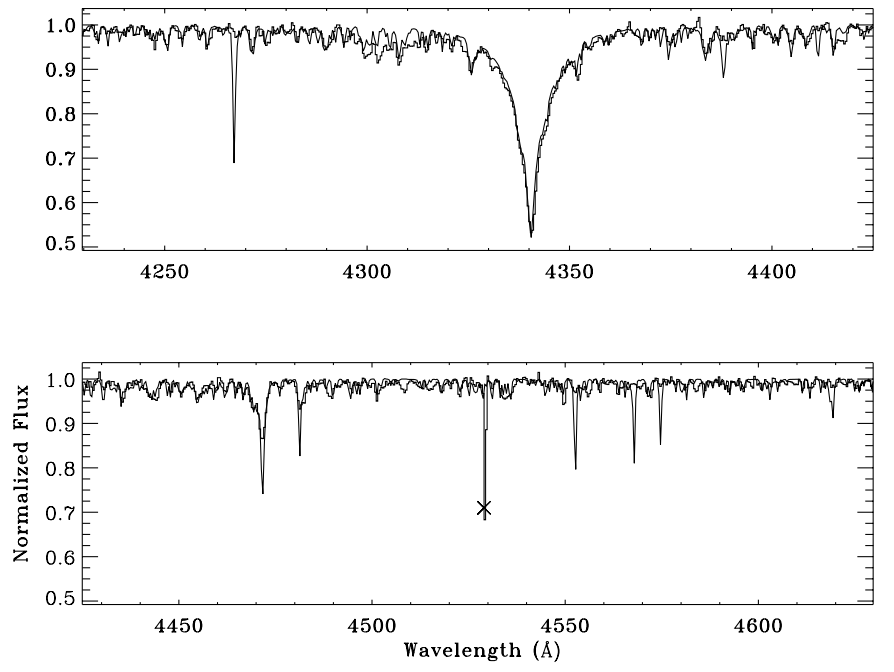

Fig. 4. As in Fig. 1 but for the composite sdB star PG 2148+095.

\section{3. $\left(\log g-T_{\text {eff }}\right)$ diagram and helium abundances}

Figure 5 compares the sdB stars analysed here with an homogeneous sample of sdB stars (Moehler, private communication) and the location of the helium main sequence (He-MS) and zero-age extreme horizontal branch (ZAEHB) (Moehler et al. 1990a). The surface gravities of hot stars in composite sdBs lie in the range $4.80 \leq$ $\log g \leq 6.00$, while our sample of single sdB stars have $4.35 \leq \log g \leq 6.25$. Although both $\log g$ and $T_{\text {eff }}$ ranges for composite sdBs are slightly smaller than for the singlespectrum stars, there is essentially no difference between their distribution in the $\log g-T_{\text {eff }}$ diagram and that of larger samples of sdB stars analyzed previously.

A striking result of this study is that the majority of single-spectrum sdB stars have helium abundances of $y=0.01$ or higher, while the composite stars have $y<0.01$ (the minimum currently available in our model grid).

Low surface He abundances are expected in sdB stars because of the competition between gravitational settling and radiative levitation acting on different ions. The same diffusive processes may be responsible for the apparently low abundances of carbon, silicon and magnesium in our sample (cf. Bergeron et al. 1988).

It has already been noted that sdB stars with composite spectra and, hence, F-, G- or K-type companions form a distinct group from those with no or unseen companions (Saffer et al. 2001). With a separate evolutionary history, a distinct surface abundance might be anticipated, but remains to be explained.

Two single-spectrum sdB stars (PG0229+064 and PG 0240+046) have $y \gg 0.01$. It is interesting that these particular examples lie at the extremities of our sample. Recalling the three groups of sdBs introduced earlier (Saffer et al. 2001), such helium-rich sdBs may form a completely separate subgroup. They were identified in the PG survey (Green et al. 1986: spectral classes sdB-O, sdOA and sdOD) and subsequently (Moehler et al. 1990a:

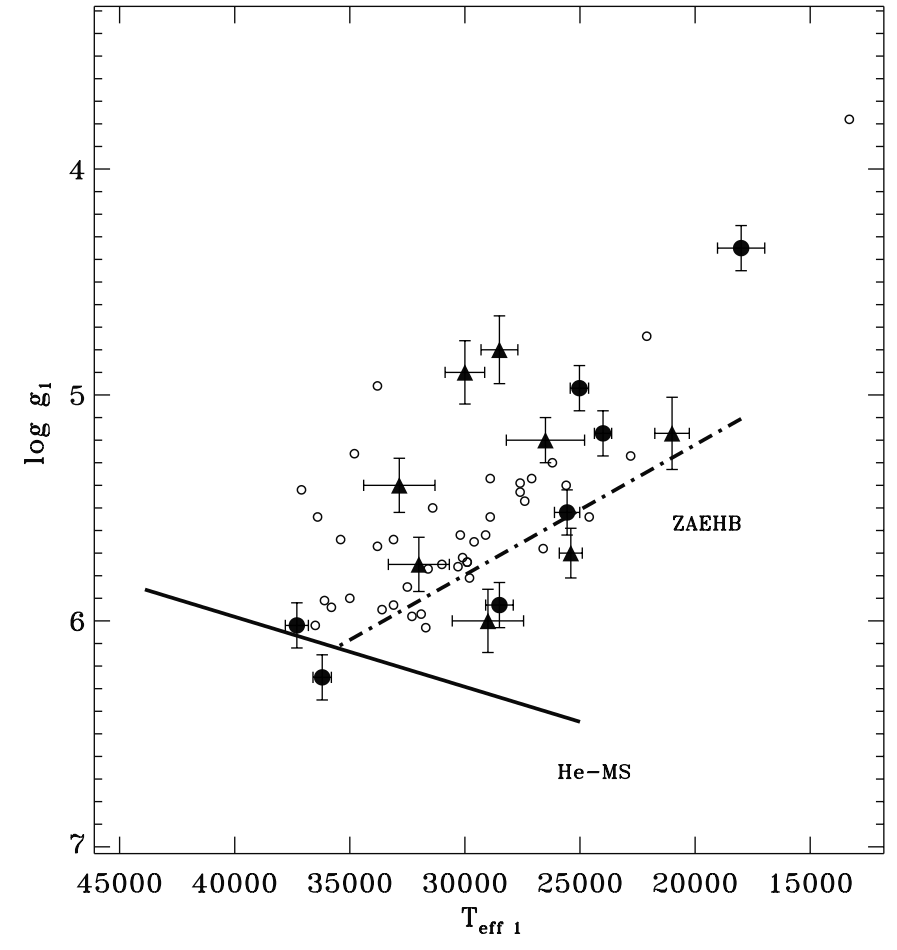

Fig. 5. Position of single sdB stars (filled circles) and composite sdB stars (filled triangles) in the $\left(\log g_{1}-T_{\text {eff } 1}\right)$ diagram as derived from the spectral analysis SFIT. Open circles represent the position of an homogeneous sample of sdB stars (Maxted et al. 2001). The position of the He-MS and ZAEHB are represented as solid and dash-dotted lines, respectively (Moehler et al. 1990a).

HesdB, Saffer et al. 1994). The latter found most of the Herich sdBs to have $T_{\text {eff }}>30000 \mathrm{~K}$, and commented that it was difficult to reconcile these stars with time-dependent diffusion calculations.

We do not currently know whether any He-rich sdB stars are members of short-period binary systems. The latter is particularly important - one scenario for the production of sdBs is the merger of two helium-white dwarfs (Iben 1990; Saio \& Jeffery 2000). The surface layers of the product may be so helium-rich that diffusive processes could not completely remove the surface helium. A significant number of He-rich binary sdBs would demand an alternative explanation.

\subsection{Composite $s d B$ companions in the HR diagram}

The cool companions in binary sdB stars have surface gravities in the range $4.30 \leq \log g_{2} \leq 4.58$. Figure 6 shows the position of the cool companion in binary sdB stars in the $\left(\log g-T_{\text {eff }}\right)$ diagram as derived from SFIT together with the location of the ZAMS and TAMS from stellar models with solar composition (Girardi et al. 2000). The observations are consistent with surface gravities of mainsequence stars. This comparison may be taken further by assuming a canonical $0.5 M_{\odot}$ for the masses of the $\mathrm{sdB}$ stars. Using the sdB surface gravities and the measured 
Table 7. Spectroscopically determined luminosities and masses for composite sdB star companions compared with the photometric analysis (Paper I). The luminosities assume 0.5 $M_{\odot}$ for the sdB stars (Heber et al. 1984; Heber 1986).

\begin{tabular}{|c|c|c|c|c|}
\hline Star & \multicolumn{2}{|c|}{ SFIT } & \multicolumn{2}{c|}{ Paper I } \\
& $L_{2} / L_{\odot}$ & $T_{\text {eff } 2} / \mathrm{K}$ & $L_{2} / L_{\odot}$ & $T_{\text {eff } 2} / \mathrm{K}$ \\
\hline 1 PG 0110+262 & $0.64 \pm 0.40$ & $5250 \pm 800$ & $0.19 \pm 0.04$ & $5485 \pm 200$ \\
2 PG 0749+658 & $0.19 \pm 0.08$ & $5000 \pm 500$ & $0.18 \pm 0.07$ & $5600 \pm 300$ \\
3 PG 1104+243 & $2.85 \pm 1.78$ & $6400 \pm 1000$ & $4.90 \pm 0.77$ & $5735 \pm 150$ \\
4 PG 1701+359 & $0.21 \pm 0.14$ & $6000 \pm 1000$ & $0.48 \pm 0.10$ & $6450 \pm 230$ \\
5 PG 1718+519 & $0.21 \pm 0.06$ & $5200 \pm 400$ & $1.02 \pm 0.15$ & $5925 \pm 70$ \\
6 PG 2110+127 & $1.45 \pm 0.44$ & $5400 \pm 400$ & $0.03 \pm 0.02$ & $5500 \pm 575$ \\
7 PG 2135+045 & $1.17 \pm 0.48$ & $5000 \pm 500$ & $0.31 \pm 0.54$ & $4375 \pm 1790$ \\
8 PG 2148+095 & $1.47 \pm 0.44$ & $5700 \pm 400$ & $0.11 \pm 0.03$ & $4375 \pm 200$ \\
\hline
\end{tabular}

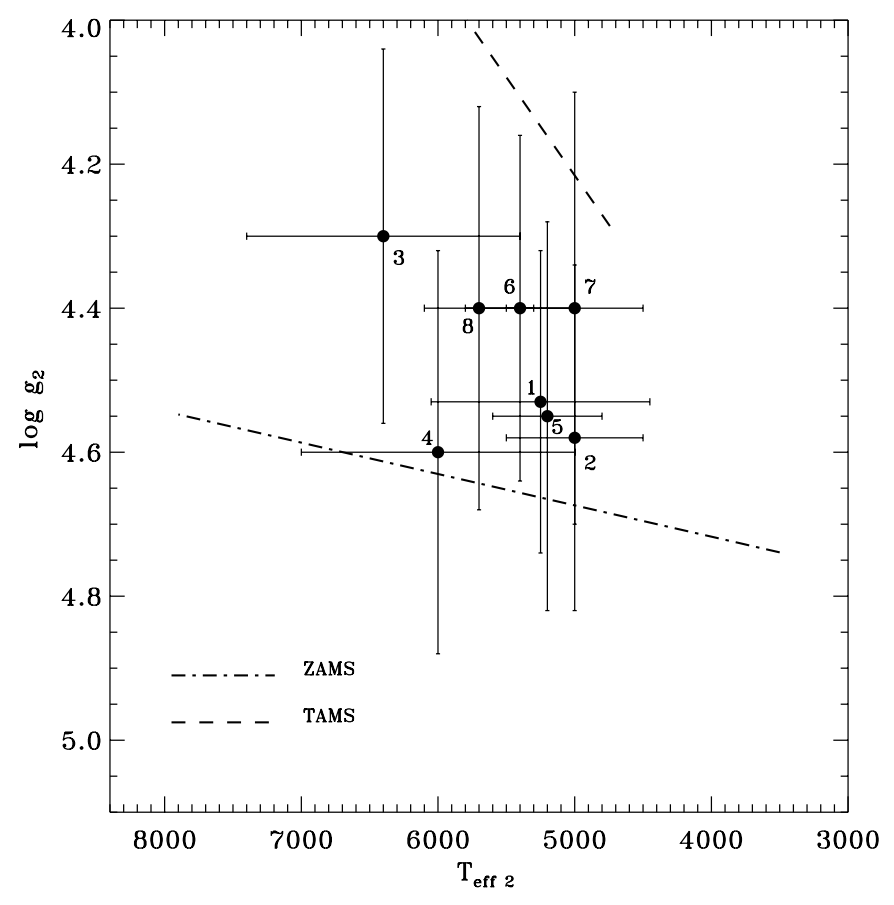

Fig. 6. Position of the cool companion in composite sdB stars in the ( $\left.\log g_{2}-T_{\text {eff } 2}\right)$ diagram as derived from the spectral analysis. The position of the ZAMS and TAMS from stellar models with solar composition (Girardi et al. 2000) are plotted as dash-dotted and dashed lines, respectively. Labels refer to identification numbers in Table 7.

radius ratios, the luminosities of both components can be calculated (Table 7).

Table 7 presents the luminosities, effective temperature and masses of the cool companions of composite sdB stars. These results reinforce our conclusion (Paper I) that the cool companions in composite sdB systems are mainsequence stars with $M \sim 1.0 \pm 0.1 M_{\odot}$.

\subsection{Mass ratios in composite $s d B$ systems}

The mass ratio of a binary system containing a hot $\mathrm{sdB}$ star and a cool companion is given by $q=M_{2} / M_{1}$. From $g=(G M) / R^{2}$ and the radius ratio $R_{2} / R_{1}$, the mass ratio can be expressed as

$q=\left(R_{2} / R_{1}\right)^{2}\left(g_{1} / g_{2}\right)$
This method of measuring $q$ is subject to the normally quite large errors in measuring $\log g$. Mass ratios for composite systems analysed by us lie in the range $0.52<$ $q<3.83$.

Assuming the cool companions in our sample to be main-sequence stars with effective temperatures $4500<$ $T_{\text {eff } 2} / \mathrm{K}<6500$, then their masses should be in the range $0.75 \leq M / M_{\odot} \leq 1.32$ (Gray 1992). Hence, assuming that the hot components of the binary systems are sdB stars with typical masses of $\sim 0.5 M_{\odot}$ (Heber et al. 1984; Heber 1986 ), then the mass ratios should be in the range $1.50 \leq$ $q \leq 2.65$. Clearly, the surface gravity ratio method is not yet sufficiently sensitive to yield the mass ratio directly.

\section{Discussion}

Green et al. (2001) have discussed the evolutionary origin of subdwarf B stars in view of findings regarding the distribution of binary periods and companions found in other surveys (Saffer et al. 2001). They deduce that sdB stars with spectral lines from a cooler companion invariably have periods longer than a year, while very shortperiod sdBs have essentially invisible companions. The deduction is that both groups are produced by Roche lobe overflow/mass transfer from low-mass stars near the tip of the red giant branch. If the initial binary is sufficiently wide and the secondary is sufficiently massive and able to accept the dynamic mass transfer of the first couple of tenths of a solar mass without filling its Roche lobe, then the initial mass ratio may be reduced sufficiently to allow stable mass transfer and avoid a common-envelope phase. In this case the orbital period would remain long, and the secondary would increase in mass, becoming a blue straggler (BS) with $\gtrsim 1 M_{\odot}$, as observed by ourselves. An important question will be to determine accurately the upper and lower limits on both masses and periods for such sdB+BS binaries. Green \& Liebert (2001) suggest that binaries with less massive secondaries would form a common-envelope and end up either merging or as shortperiod sdB+MS systems.

The division of sdBs into long- and short-period binaries suggests a reason for the difference in helium abundance between the two samples. While low-helium (and -metal) abundances are known to be a result of atmospheric diffusion in high-gravity stars within a certain 
temperature range, external forces may partially disrupt these. Tidal interaction due to a binary companion will be much stronger in a short-period than in a long-period system. Unless the sdB star is rotating completely synchronously, tidal effects will operate on timescales shorter than diffusion $\left(\sim 10^{5}\right.$ years $)$ and may dilute the chemical separation. Extremely low-hydrogen abundances would therefore be seen preferentially in long-period sdB binaries.

The presence of sdBs within the sample with helium abundances significantly greater than normal (e.g. PG 0229+064, PG 0240+046) may be a consequence of their belonging to sdB group (1) - apparently single stars (Saffer et al. 2001). It is interesting that no sdB star with $y>0.03$ is known to be a short-period binary (Maxted et al. 2001). Since sdB stars are known with extremely high helium abundances (cf. Jeffery et al. 1987), we suggest that these could have an entirely separate origin, being the products of helium plus helium white dwarf mergers (Iben 1990). Evidence for such a conclusion is provided by the extreme helium star V652 Her (Jeffery et al. 2001), considered to be strong evidence for such a merger product evolving to become an isolated helium main-sequence star (Saio \& Jeffery 2000). When it becomes a subdwarf, diffusion will inevitably modify the initially helium-rich atmosphere. However, with a much more limited reservoir of hydrogen, extremely low helium abundances will be difficult to achieve. Consequently sdBs produced by mergers could be expected to show a very wide range of helium abundances.

\section{Conclusions}

We have analysed representative samples of $\mathrm{sdB}$ stars having apparently single or composite spectra. The atmospheric properties of the $\mathrm{sdB}$ stars were measured by comparing moderate-resolution blue spectra with theoretical models. In the case of sdBs with composite spectra, the atmospheric properties of the cool companions were measured from the blue spectra and from near-infrared spectra, where the infrared calcium triplet provides an invaluable surface gravity indicator.

Both samples covered a similarly wide range in $T_{\text {eff }}$ and $\log g$. However the composite sdB stars invariably have lower helium abundances in their atmospheres than the single-spectrum sdBs. Although we cannot entirely explain this phenomenon, we suggest that it may be due to tidal effects disrupting diffusive separation in short-period systems more than in long-period systems. Some of the composite sdB stars also showed depletions of metals including silicon, carbon and magnesium.

Assuming a typical surface luminosity representing all subdwarf B stars of $\log \left(L_{\mathrm{sdB}} / L_{\odot}\right)=1.40 \pm 0.13$, the majority of the companions of composite sdBs have luminosities in the range $0.4 \leq L_{\mathrm{cool}} / L_{\odot} \leq 2.6$, consistent with being main-sequence stars of about $1 \pm 0.2 M_{\odot}$. This supports the hypothesis that composite sdB stars are the result of Roche lobe overflow near the red-giant tip in a low-mass binary with nearly equal initial masses (Green et al. 2001).

This is the first time that an attempt has been made to model accurately the spectra of composite subdwarf B stars and to measure the cool star luminosity using the infrared calcium triplet. Although successfully executed, higher resolution spectra will be needed to measure the metallicity of the cool star and hence the radius ratios with greater accuracy.

Acknowledgements. This research is supported by a grant to the Armagh Observatory from the Northern Ireland Department of Culture, Arts and Leisure and by the UK Particle Physics and Astronomy Research Council through the award of telescope time and travel grants. We are particularly grateful to Prof Philip Dufton and Drs Sabine Moehler and Betsy Green for helpful discussions and to Dr Don Pollacco for obtaining some of the observations.

\section{References}

Allard, F., Wesemael, F., Fontaine, G., Bergeron, G., \& Lamontagne, R. 1994, AJ, 197, 1565

Aznar Cuadrado, R. 2001, Ph.D. Thesis, Queen's University of Belfast

Aznar Cuadrado, R., \& Jeffery, C. S. 2001, A\&A, 368, 994 (Paper I)

Bergeron, P., Wesemael, F., Michaud, G., \& Fontaine, G. 1988, ApJ, 332, 964

Brown, T. M., Ferguson, H. C., Davidsen, A. F., \& Dorman, B. 1997, ApJ, 482, 685

Cohen, J. G. 1979, ApJ, 228, 405

D'Cruz, N. L., Dorman, B., Rood, R. T., \& O'Connell, R. W. 1996, ApJ, 466, 359

Ferguson, D. H., Green, R. F., \& Liebert, J. 1984, ApJ, 287, 320

Girardi, L., Bressan, A., Bertelli, G., \& Chiosi, C. 2000, A\&AS, 141,371

Green, R. F., Schmidt, M., \& Liebert, J. 1986, ApJS, 61, 305

Green, E. M., Liebert, J., \& Saffer, R. A. 2001, Proceedings of the Twelth European Conference on White Dwarf Stars, ed. H. L. Shipman, J. L. Provencal, J. MacDonald, \& S. Goodchild, ASP Conf. Ser., 226

Gray, D. F. 1992, The observation and analysis of stellar photospheres (Cambridge University Press, Cambridge)

Hardorp, J., \& Scholz, M. 1970, ApJS, 19, 193

Heber, U. 1986, A\&A, 155, 33

Heber, U., Hunger, K., Jonas, G., \& Kudritzki, R. P. 1984, A\&A, 130, 119

Heber, U., Edelmann, H., Lemke, M., Napiwotzki, R., \& Engels, D. 1999, 11th European Workshop on White Dwarfs, ed. S. E. Solheim, \& E. G. Meistas (Astronomical Society of the Pacific), ASP Conf. Ser., 169, 551

Iben, I., Jr. 1990, ApJ, 353, 215

Jeffery, C. S. 1998, MNRAS, 294, 391

Jeffery, C. S. 1991, Newsletter on Analysis of Astronomical Spectra, 16, 17

Jeffery, C. S., \& Aznar Cuadrado, R. 2001, A\&A, 378, 936

Jeffery, C. S., \& Heber, U. 1992, A\&A, 260, 133

Jeffery, C. S., \& Pollacco, D. L. 1998, MNRAS, 298, 179

Jeffery, C. S., Lester, J., \& Short, I. 1996, CCP7 Newsletter, 24,13 
Jeffery, C. S., Drilling, J. S., Harrison, P. M., Heber, U., \& Moehler, S. 1997, A\&AS, 125, 501

Jeffery, C. S., Hamill, P. J., Harrison, P. M., \& Jeffers, S. V. 1998, A\&A, 340, 476

Jeffery, C. S., Woolf, V. M., \& Pollacco, D. L. 2001, A\&A, 376, 497

Jones, J. E., Alloin, D. M., \& Jones, B. J. T. 1984, ApJ, 283, 457

Jørgensen, U. G., Carlsson, M., \& Johnson, H. R. 1992, A\&A, 254,258

Kodaira, K., \& Scholz, M. 1970, A\&A, 6, 93

Kurucz, R. L. 1979, ApJS, 40, 1

Kurucz, R. L. 1991, in Stellar Atmospheres: Beyond Classical Models, ed. L. Crivellari, I. Hubeny, \& D. G. Hummer, NATO ASI Series Vol. C341 (Kluwer, Dordrecht), 441

Kurucz, R. L. 1993a, Kurucz CD-ROM No. 13. Cambridge, Mass.: Smithsonian Astrophysical Observatory

Kurucz, R. L. 1993b, Kurucz CD-ROM No. 18. Cambridge, Mass.: Smithsonian Astrophysical Observatory

Lamontagne, R., Wesemael, F., Fontaine, G., \& Sion, E. M. 1985, ApJ, 299, 496

Lamontagne, R., Wesemael, F., \& Fontaine, G. 1987, ApJ, 318, 844

Lang, K. R. 1992, in Astrophysical data: Stars and planets (Springer-Verlag, New York)

Maxted, P. F. L., Heber, U., Marsh, T. R., \& North, R. C. 2001, MNRAS, 326, 1391
Moehler, S., Heber, U., \& Boer, K. S. 1990a, A\&A, 239, 265

Moehler, S., Heber, U., \& Rupprecht, G. 1997, A\&A, 319, 109

Moehler, S., Richtler, T., Boer, K. S., Dettmar, R. J., \& Heber, U. 1990b, A\&AS, 86, 53

Peters, G. J. 1976, ApJS, 30, 551

Press, W. H., Flannery, B. P., Teukolsky, S. A., \& Vetterling, W. T. 1989, Numerical Recipes: The Art of Scientific Computing (Cambridge University Press)

Ramspeck, M., Heber, U., \& Edelmann, H. 2001, A\&A, 378, 907

Saio, H., \& Jeffery, C. S. 2000, MNRAS, 313, 671

Saffer, R. A., Bergeron, P., Koester, D., \& Liebert, J. 1994, ApJ, 432, 351

Saffer, R. A., Green, E. M., \& Bowers, T. P. 2001, Proceedings of the Twelth European Conference on White Dwarf Stars, ed. H. L. Shipman, J. L. Provencal, J. MacDonald, \& S. Goodchild, ASP Conf. Ser., 226

Smith, G., \& Drake, J. J. 1987, A\&A, 181, 103

Theissen, A., Moehler, S., Heber, U., \& de Boer, K. S. 1993, A\&A, 273, 524

Theissen, A., Moehler, S., Heber, U., Schmidt, J. H. K., \& de Boer, K. S. 1995, A\&A, 298, 577

Thejll, P., Bauer, F., Saffer, R., et al. 1994, ApJ, 433, 819

Thejll, P., Ulla, A., \& MacDonald, J. 1995, A\&A, 303, 773

Ulla, A., \& Thejll, P. 1998, A\&AS, 132, 1 